\title{
The Development of Bullous Pemphigoid in a Known Case of Psoriasis Vulgaris: a case report
}

Yousef Abuhaimed $^{1}$, Alaa Salem Alshamsy ${ }^{1}$, Sarah Hasan Albreiki ${ }^{2}$, Khalid Saleh Alkarawi ${ }^{2}$

${ }^{1}$ King Fahad University Hospital, Khobar, Saudi Arabia

${ }^{2}$ MD, Consultant Dermatologist in King Fahad University Hospital, Khobar, Saudi Arabia

\section{Type of article: Case report}

\begin{abstract}
Introduction: The development of Bullous pemphigoid (BP) in a patient with Psoriasis vulgaris (PV) is rare, as Bullous pemphigoid affects a different age group and its cause is not related to psoriasis. The cause of coexistence between the two diseases is still not clear and requires more reporting and review.

Case Presentation: Here, we report a case of a 55-year-old woman with a 22 -year history of Psoriasis vulgaris who developed itching and blisters to all four limbs around the scaly plaques of PV. Biopsy of the lesion revealed sub-epidermal bullae and an eosinophilic inflammatory infiltrate. Immunofluorescence microscopy showed linear C3 deposition at the basement membrane zone, and the condition was diagnosed as Bullous pemphigoid. Treatment in such conditions varies among reported cases, and includes methotrexate, cyclosporine, dapsone, azathioprine, and others. In this case, methotrexate was suggested but because patient could not tolerate the course of methotrexate she was started on a combination therapy of cyclosporine $100 \mathrm{mg}$ PO twice daily and tetracycline PO $500 \mathrm{mg}$ PO Q 6 hourly. Both conditions showed significant improvement within 4 weeks.

Take-away lesson: As the cause of the concurrence is still unclear, the correct management for such cases still vary. The case report highlighted our experience to benefit dermatologists and physicians, with one therapeutic regimen that can benefit patients who cannot tolerate methotrexate due to its side effects.
\end{abstract}

Keywords: Psoriasis, Psoriasis vulgaris, Pemphigoid, Bullous pemphigoid, Linear C3 Deposition

Note: This case report is prepared using the CARE Checklist (2013) of information to include when writing a case report (https://www.care-statement.org). The CARE guidelines for case reports help reduce bias, increase transparency, and provide early signals of what works, for which patients, and under which circumstance.

\section{Introduction}

Bullous pemphigoid is a known skin disease characterized by the eruption of tense subepidermal bullae. It affects mostly elderly people over 70 years of age (1), whereas Psoriasis vulgaris is a chronic inflammatory skin condition that is characterized by the emergence of scaly plaques on the skin (2). The concurrence of the two diseases in one patient is a rare event, and the pathogenic relation between them is still unclear (3). No other cases of such association were reported in Saudi Arabia. It sheds light on a different presentation and age group for Bullous pemphigoid among patients with psoriasis within the population of Saudi Arabia and worldwide which can be of clinical significance if the association between the two diseases was understood. In reported cases, different treatment modalities were used including methotrexate, cyclosporine, dapsone, azathioprine, and many others. It was necessary to approach our case differently, as our patient could not tolerate methotrexate and we had to look into alternatives. A combination therapy of Cyclosporine and Tetracycline was used and it showed significant improvements in both conditions. The value of our case involves highlighting a different treatment regimen for a

\section{Corresponding author:}

Yousef Abuhaimed, King Fahad University Hospital, Khobar, Saudi Arabia.

Tel: +966138683458, Email: YousefAbuhaimed@gmail.com

Received: January 04, 2019, Accepted: February 07, 2019, Published: March 2019

iThenticate screening: February 07, 2019, English editing: February 09, 2019, Quality control: February 12, 2019

This article has been reviewed / commented by three experts

Ethics approval: King Fahd University Hospital (Ref: 2025418985)

(C) 2019 The Authors. This is an open access article under the terms of the Creative Commons Attribution-NonCommercialNoDerivs License, which permits use and distribution in any medium, provided the original work is properly cited, the use is non-commercial and no modifications or adaptations are made. 
rare association between two dermatological diseases for patients who could not tolerate methotrexate as a management for their condition.

\section{Case presentation}

\subsection{Patient information}

The patient was a 55-year-old female (white, homemaker) who was a known case of generalized Psoriasis vulgaris for more than 22 years, mild systolic hypertension, and dyslipidemia. She presented at the dermatology department at King Fahd University hospital in Khobar, Kingdom of Saudi Arabia for follow up regarding her psoriasis where it was noted that the patient had developed multiple blisters over her limbs that were causing itchiness and discomfort (Figure 1).

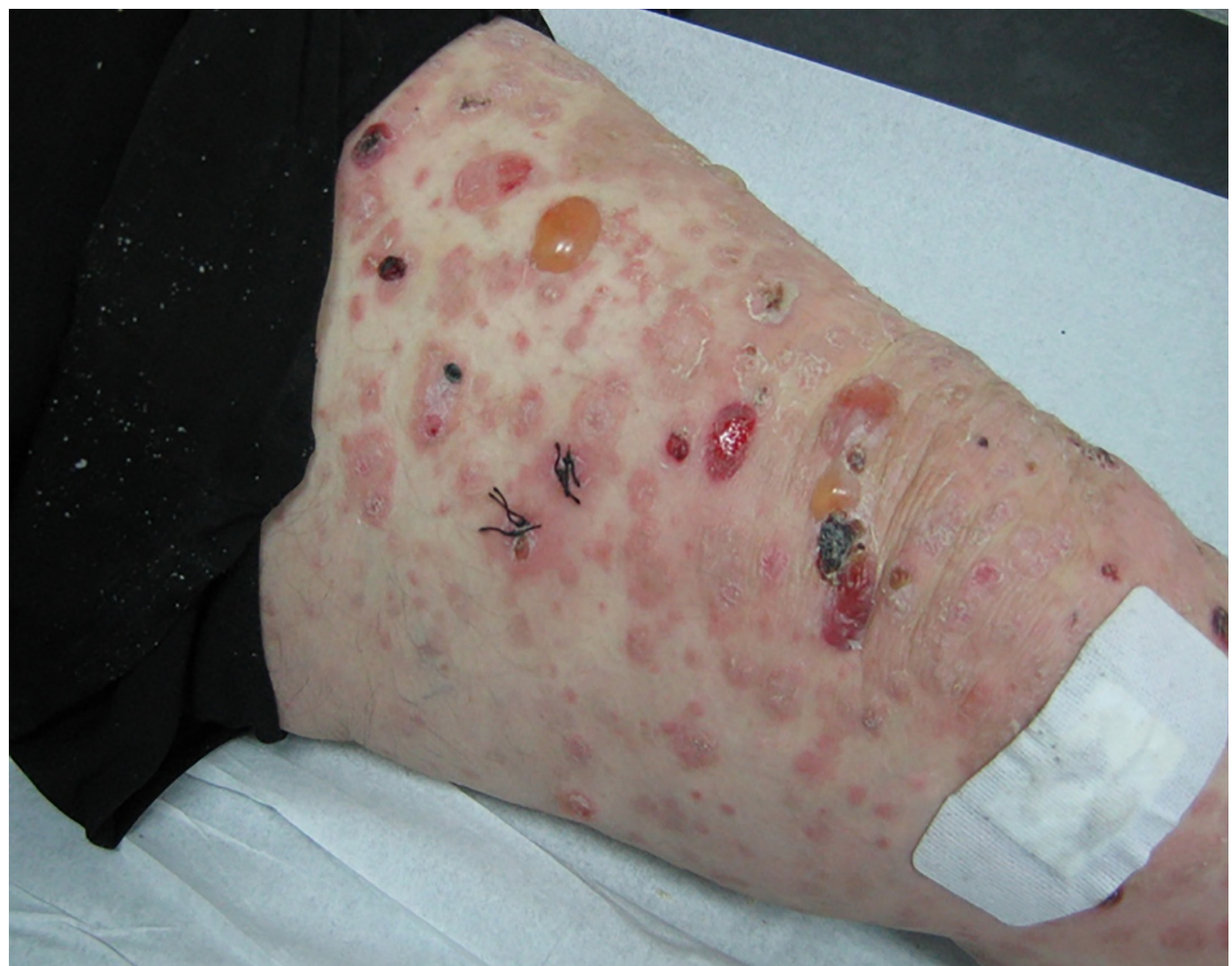

Figure 1. tense blisters and plaques of psoriasis occurring simultaneously in the patient.

\subsection{Clinical Findings, relevant past interventions and their outcome}

The psoriasis was controlled by topical treatments (calcipotriol cream, clobetasol propionate ointment and Tar and Salicylic acid shampoo) until her condition began to deteriorate and her psoriatic lesions covered more than $60 \%$ of her body surface area. Thereupon she was started on cyclosporine at a dose of $150 \mathrm{mg}$ twice daily after being medically evaluated. The patient's psoriasis cleared and she was clinically free of psoriasis for about a year, after which she started complaining of a severe generalized itching, which was initially thought to be related to Psoriasis vulgaris. However, this itching was followed a month later by an eruption of tense bullae. The bullae were distributed mainly over her upper and lower limbs and to a lesser extent on the trunk with sparing of the mucous membranes.

\subsection{Diagnostic Assessment}

\subsubsection{Diagnostic methods}

A biopsy was carried out, and it revealed sub-epidermal blisters and eosinophilic inflammatory infiltrate. Immunofluorescence microscopy showed linear C3 deposition at the basement membrane zone, and she was diagnosed as a case of Bullous pemphigoid. Psoriasis lesions were also seen at the time of the eruption of bullae. 


\subsubsection{Diagnostic challenges and reasoning}

The patient was not eligible at first but due to her condition she was filed for eligibility and her request was approved. Her condition was first thought to be related to her Psoriasis, but to rule out Bullous pemphigoid, a biopsy was done and it revealed sub-epidermal blisters and eosinophilic inflammatory infiltrate. Immunofluorescence microscopy showed linear $\mathrm{C} 3$ deposition at the basement membrane zone, and she was diagnosed as a case of Bullous pemphigoid. Psoriasis lesions were also seen at the time of the eruption of bullae.

\subsubsection{Prognostic characteristics}

Both Psoriasis vulgaris and Bullous pemphigoid are chronic diseases that have multiple factors affecting the prognosis. Relapses can occur in both diseases.

\subsection{Therapeutic Intervention}

We decided to start the patient on tetracycline $500 \mathrm{mg} / 6 \mathrm{hr}$, and opted to avoid systemic steroids for the fear of flaring up her psoriasis when tapering the dose. She was still on cyclosporine to control her psoriasis, and regular monitoring of cyclosporine level was carried out. The patient showed significant improvement for both conditions. Bullous pemphigoid lesions started to resolve by the following month. Both conditions improved significantly within 4 weeks after the initiation of treatment.

\subsection{Follow-up and outcomes}

The patient's condition resolved regarding her plaques and blisters within 4 weeks while on the medication (Figure 2). As we were tapering down the medications, she developed tinnitus and vertigo, which can be caused by Tetracycline, and medication was discontinued. The patient was on follow up with the dermatology department over the following years and her blisters did not recur. For her psoriasis, she was maintained on cyclosporine and she was responding well. However, her psoriasis flared up later during that year as the patient was undergoing psychological stress due to social issues, which improved later on the same therapy.

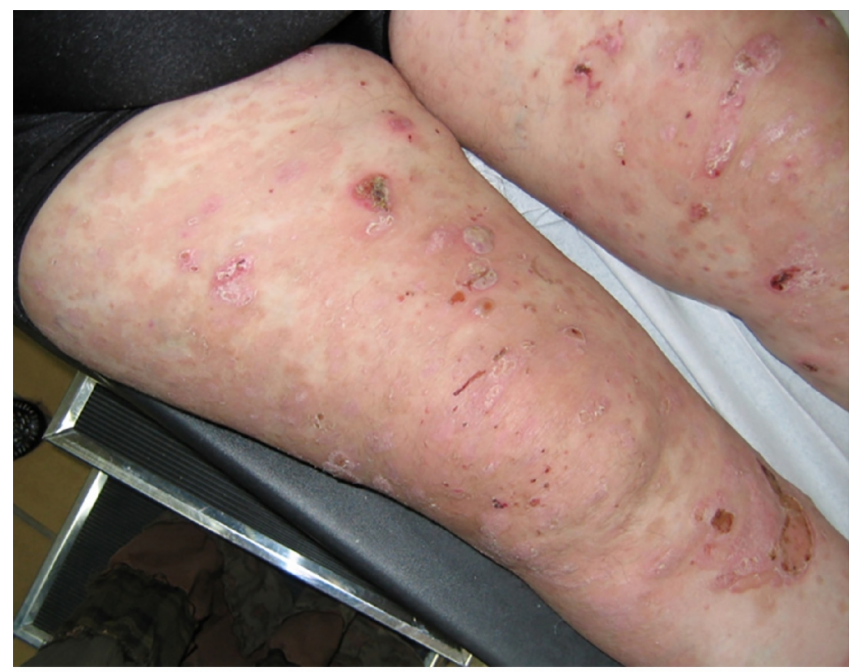

a

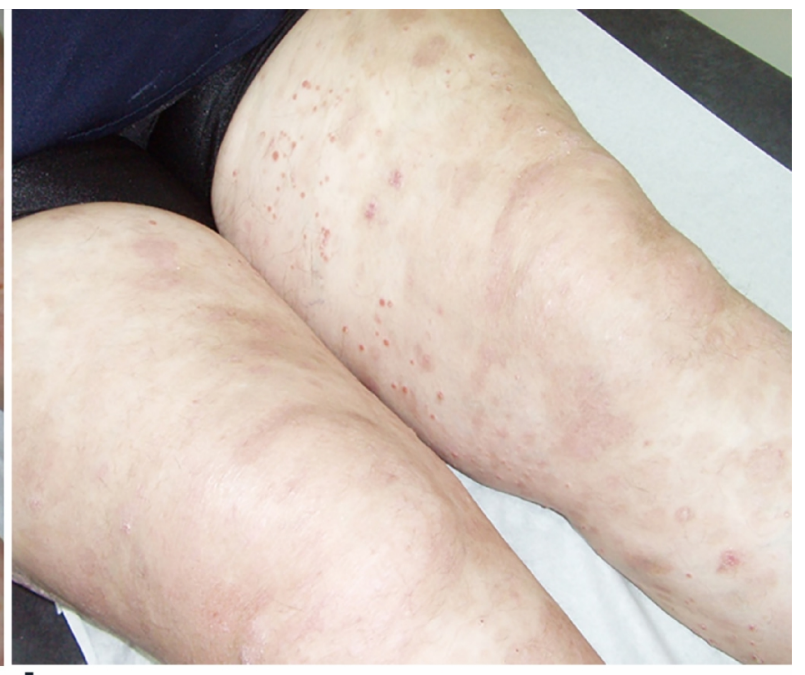

b

Figure 2. Patient improving after receiving treatment (a), and complete resolution after 4 weeks of treatment (b).

\subsection{Ethics of case report}

The Research Ethics Committee in King Fahd University Hospital approved the case study \#2025418985. A written informed consent was signed by the patient on January 22, 2019 and was attached. Patient confidentiality was maintained as nothing that can be related to the patient was written in the case report.

\section{Discussion}

The coexistence of Psoriasis and Bullous pemphigoid was first described in 1929 by Dr. Bloom where he highlighted the association of both diseases and their concomitance (4). Some literature report Psoriasis vulgaris as being linked with several other autoimmune bullous diseases (5-8). Bullous pemphigoid is the most frequent condition to be linked with psoriasis among all other bullous diseases; around 60 cases of such associations have 
been described in English literature, but it is still a rare occurrence. It was reported that this association is more common in males than females with the onset age averaging at 63 years. Psoriasis preceded the development of Bullous pemphigoid in most cases, and the average time interval between the two diseases was around 20 years (ranging from 1-60 years) (3). To the best of our knowledge, our case is the only reported case of such association in the Middle East. The exact mechanism for coexistence of both conditions is still not fully understood and was not identified in our case. However, the literature highlights that the association is not coincidental. A few theories have been suggested such as the irritating nature of some treatments of psoriasis, pathological events at the basement membrane from psoriasis itself, common immunological or immunogenetic mechanisms and triggering factors occurring simultaneously which result in precipitating bullous diseases in these patients. It is difficult to highlight a single causative factor in the development of Bullous pemphigoid in psoriasis patients as it has occurred in patients undergoing random treatment modalities, and in those who were not receiving any treatment for their condition (9).

Some case reports have highlighted the role of PUVA therapy in the development of Bullous pemphigoid in cases of psoriasis and even in non-psoriatic diseases such as vitiligo and Mycosis fungoides (10-14). Our patient underwent PUVA therapy for her psoriasis but it was discontinued around 3 years before she developed the blisters, which made the association unfavorable. However, Abel et al. suggested that PUVA triggers the autoimmune reaction de novo as it modifies the cellular DNA and nuclear proteins antigenically, it also alters basement membrane proteins, such as BP antigens, hence explaining the relationship (14).

Attention is now being set towards a possible immunological association between the two diseases. As recent studies have established that the primary pathogenetic subset of $\mathrm{T}$ cells in psoriasis is Th17 and not Th1 as was previously understood, it is identified that this subset of T cells also plays a key role in autoimmunity, but this was not studied in our case (15). Therefore, psoriasis might be predisposing the immune system in a way that, under a set of circumstances such as the dysregulation of T-cell activities, leads to autoimmune responses resulting in the release of antibodies to the antigens of the basement membrane in psoriasis patients.

Treatment in such conditions varied among the reported cases, and included methotrexate, cyclosporine, dapsone, azathioprine and others. The use of systemic steroids was avoided in such cases to avoid triggering pustular psoriasis. A course of methotrexate was suggested to our patient but it was refused as she had experienced many side effects such as blurry vision, nausea, and severe dizziness during a previous trial that was intended to control her psoriasis, and biological agents were not available for the patient. A combination therapy of cyclosporine 100 $\mathrm{mg}$ PO twice daily and tetracycline PO $500 \mathrm{mg}$ PO/6hr was initiated in our patient and both psoriasis and Bullous pemphigoid improved significantly within 4 weeks.

\section{Conclusions}

The development of Bullous pemphigoid in a patient with Psoriasis vulgaris is rare. In our case, a 55-year-old female known case of Psoriasis vulgaris developed itchy blisters on her limbs, which were diagnosed as Bullous pemphigoid by biopsy. Unlike most reported cases, we could not proceed with methotrexate as our patient could not tolerate it, and was given a combination therapy of cyclosporine and tetracycline instead, which showed significant improvement over 4 weeks. Even though the association between the two diseases was first described in 1929, the exact mechanism of association is still not fully understood. Multiple theories were highlighted in the literature but none of them were directly associated and there was no main cause identified, which calls for more future research and encourages the reporting of such cases.

\section{Acknowledgments:}

We would like to thank the patient described for allowing us to share her condition. We would also like to thank the dermatology department and King Fahd University Hospital for their efforts in the preparation of this case report.

\section{Conflict of Interest:}

There is no conflict of interest to be declared.

\section{Authors' contributions:}

All authors contributed to this project and article equally. All authors read and approved the final manuscript. 


\section{References:}

1) Wojnarowska F, Kirtschig G, Highet AS, Venning VA, Khumalo NP. Guidelines for the management of bullous pemphigoid. Br J Dermatol. 2002; 147(2): 214-21. doi: 10.1046/j.1365-2133.2002.04835.x. PMID: 12174090 .

2) Nestle FO, Kaplan DH, Barker J . Mechanisms of Disease Psoriasis. N Engl J Med. 2009; 361: $496-509$.

3) Wilczek A, Sticherling M. Concomitant psoriasis and bullous pemphigoid: coincidence or pathogenic relationship? Int J Dermatol. 2006; 45(11): 1353-7. doi: 10.1111/j.1365-4632.2006.02861.x.

4) Bloom D. Psoriasis with superimposed bullous eruption. Med J Rec. 1929; 130: 246.

5) Saraceno R, Chimenti S. Associated conditions of psoriasis. In: Chimenti S, editor. Psoriasis. Florence: See- Firenze. 2005; 105-11.

6) Grunwald MH, David M, Feuerman EJ. Coexistence of psoriasis vulgaris and bullous diseases. J Am Acad Dermatol. 1985; 13(2): 224-8. doi: 10.1016/S0190-9622(85)70163-6.

7) Endo Y, Tamura A, Ishikawa O, Miyachi Y, Hashimoto T. Psoriasis vulgaris coexistent with epidermolysis bullosa acquisita. Br J Dermatol. 1997; 137(5): 783-6. doi: 10.1111/j.1365-2133.1997.tb01119.x. PMID: 9415242.

8) Morris SD, Mallipeddi R, Oyama N, Gratian MJ, Harman KE, Bhogal BS, et al. Psoriasis bullosa acquisita. Clin Exp Dermatol. 2002; 27(8): 665-9. doi: 10.1046/j.1365-2230.2002.01100.x. PMID: 12472542.

9) Kirtschig G, Chow ETY, Venning VA, Wojnarowska FT. Acquired subepidermal bullous diseases associated with psoriasis: A clinical, immunopathological and immunogenetic study. Br J Dermatol. 1996; 135(5): 738-45. doi: 10.1111/j.1365-2133.1996.tb03883.x. PMID: 8977674.

10) Perl S, Rappersberger K, Födinger D, Anegg B, Hönigsmann H, Ortel B. Bullous pemphigoid induced by puva therapy. Dermatology. 1996; 193(3): 245-7. doi: 10.1159/000246255. PMID: 8944349.

11) George PM. Bullous pemphigoid possibly induced by psoralen plus ultraviolet A therapy. Photodermatol Photoimmunol Photomed. 1995; 11(5-6): 185-7. doi: 10.1111/j.1600-0781.1995.tb00166.x.

12) Patterson JW, Ali M, Murray JC, Hazka JA. Bullous Pemphigoid: Occurrence in a Patient with Mycosis Fungoides Receiving PUVA and Topical Nitrogen Mustard Therapy. Int J Dermatol. 1985; 24(1): $173-6$. doi: 10.1111/j.1365-4362.1985.tb05416.x. PMID: 3888880.

13) Riyaz N, Nasir N, Bindu V, Sasidharanpillai S. Bullous pemphigoid induced by topical PUVASOL. Indian Journal of Dermatology, Venereology and Leprology. 2014; 80(4): 363-4. doi: 10.4103/03786323.136936.

14) Abel EA, Bennett A. Bullous pemphigoid. Occurrence in psoriasis treated with psoralens plus long-wave ultraviolet radiation. Arch Dermatol. 1979; 115(8): 988. PMID: 380470.

15) Griffiths CE, Barker JN. Psoriasis. In: Burns T, Breathnach S, Cox N, Griffiths C, editors. Rook's Textbook of Dermatology. 8th ed. Oxford: Wiley Blackwell; 2010; 1-20.60. doi: 10.1002/9781444317633.ch20. 